\title{
Plantar Pressures in Rheumatoid Arthritis Using Prefabricated Metatarsal Padding
}

\author{
Lorraine Jackson, BSc(Hons)* \\ Jodi Binning, MSc† \\ Julia Potter, PhD¥
}

We sought to determine whether one of two prefabricated insole designs could better manage high forefoot plantar pressures in patients with rheumatoid arthritis. Ten subjects with rheumatoid arthritis who experienced pain with shod weightbearing were studied by using a plantar pressure measurement system. Two insole designs and a shoe-only control condition were randomly tested in repeated trials. Dome- and bar-shaped metatarsal pads made of latex foam were incorporated into full-length insoles made of urethane. Significant reductions in mean peak plantar pressures over the central metatarsals were noted when using the insole and dome pad design (12\% [33 kPa]) and the insole and bar pad design $(21 \%$ [58 kPa]) compared with the shoe-only condition. A prefabricated insole design incorporating a bar metatarsal pad is recommended to manage high forefoot plantar pressures in patients with rheumatoid arthritis. (J Am Podiatr Med Assoc 94(3): 239-245, 2004)

Patients with established rheumatoid arthritis often bear a disproportionate amount of body weight over the metatarsal heads. ${ }^{1}$ As joint deformity progresses, the plantar fat pad may be displaced, with a loss of cushioning of the forefoot and associated metatarsalgia. ${ }^{2}$ Abnormal gait with reduced mobility may ensue with the development of pressure lesions, a splay forefoot, and bursae formation. ${ }^{3-7}$ Management of the

*Southampton City PCT, Podiatry Services, Central Health Clinic, Southampton, England. Ms. Jackson is now with the NHS Direct Hampshire \& Isle of Wight, Hedge End, Southampton, England.

$\dagger$ New Generation Project, Southampton General Hospital, Southampton, England.

$\$$ Podiatry Research Group, University of Southampton, Highfield, Southampton, England.

Corresponding author: Lorraine Jackson, BSc(Hons), NHS Direct Hampshire \& Isle of Wight, Strawberry Fields, Berrywood Business Village, Tollbar Way, Hedge End, Southampton, Hampshire SO30 2UN, England. deformed rheumatoid arthritis foot includes conservative and surgical interventions; Craxford et $\mathrm{al}^{8}$ found that recurrence rates of metatarsalgia were high after surgery and that there was little difference in the outcomes of operative and nonoperative treatments. Promising research for individuals with early forefoot disease has identified an important interrelation between rearfoot position and forefoot pressure. ${ }^{9}$ Veves et al $^{10}$ reported positive results after studying the effects of padded hosiery with added density at the forefoot region, although it was acknowledged that such hosiery may be cosmetically unacceptable to some patients. In an attempt to support joint function and reduce pressure and possibly pain, the metatarsal pad has been developed. ${ }^{11-13}$ Metatarsal padding offers immediate treatment, with the explicit aim of distributing forces more equally over the plantar surface of the foot. ${ }^{14,15}$ Numerous types of plantar padding 
are available; some are molded into a prescription insole, and others are separate prefabricated devices manufactured from a variety of materials in various shapes and sizes. The success of metatarsal pads is evident in the national audit by Yates, ${ }^{16}$ in which 55 (31\%) of 176 podiatric physicians in the United Kingdom reported that they regularly prescribed them. Unfortunately, the available choices are overwhelming, making clinical protocols based on scientific evidence difficult to establish. The prudent course of action, therefore, may be to isolate single groups, such as patients with rheumatoid arthritis, to provide the clinical evidence. ${ }^{4,17}$

Several studies ${ }^{18-21}$ have considered the use of prefabricated metatarsal pads and plantar pressures in asymptomatic subjects. Holmes and Timmerman ${ }^{18}$ selected a small metatarsal pad affixed directly to the foot and assessed single-step peak pressure data in ten subjects. Although the authors found that peak pressures were reduced by $12 \%$ to $60 \%$ in seven subjects, concluding that metatarsal pads were an effective means of reducing forefoot pressures, the study was limited in that the properties of the "soft metatarsal pad" were not stated. When Hayda et al ${ }^{20}$ studied the effects of three different types of metatarsal pads on plantar pressures in ten subjects, they determined that the position and the material properties were crucial, and they recommended that a small felt pad be positioned $5 \mathrm{~mm}$ distal to the metatarsal heads. This pad decreased average pressures by $19 \%$ at the metatarsal heads.

More recently, Kelly and Winson ${ }^{22}$ studied subjects with metatarsalgia, sampling single-step data from a Musgrave Footprint pressure plate (Musgrave Systems Ltd, Llangollen, North Wales). They taped ready-made insoles of two designs to the subjects' feet; one design incorporated a metatarsal dome in a fixed position, and the other permitted partial customization with padding. During the 8-week trial, two groups (46 feet) were assessed for pain and plantar pressures. The customized insoles yielded better results in terms of forefoot pressure reduction compared with the barefoot condition $(P<.001)$.

Hodge et $\mathrm{al}^{15}$ investigated four different orthoses in 12 subjects with rheumatoid arthritis. One of the insoles studied was prefabricated without any metatarsal padding, and the remaining orthoses were custom-molded, two with the addition of metatarsal pads. Use of the Pedar in-shoe pressure-measurement system (Novel GmbH, Munich, Germany) facilitated the analysis of consecutive footsteps and revealed that all of the orthoses significantly reduced pressure beneath the first and second metatarsal heads compared with shoes only $(P<.05)$. A dome design on a customized insole was the most effective orthosis in terms of reducing subjective pain ratings $(P<.05)$.

To date, no study has compared pressures when using a prefabricated insole and prefabricated metatarsal pads in patients diagnosed as having rheumatoid arthritis. The aim of this research was to determine whether a prefabricated insole and metatarsal pad of a specific design can manage plantar pressures more effectively than another design.

\section{Materials and Methods}

Following local ethics committee approval, an opportunistic subject sample was recruited from the Southampton Podiatry Centre in Southampton. All subjects gave written consent to participate in the study and visited the clinical center once for data collection.

\section{Subjects}

Of 15 subjects invited to participate, 11 were recruited. All participants had a history of rheumatoid arthritis, as defined by the American Rheumatism Association. ${ }^{23}$ Inclusion criteria were a diagnosis of rheumatoid arthritis, pain with shod weightbearing in the forefoot region, and the ability to understand the procedure. Subjects were excluded if they had previous excision of one or more metatarsal heads, excessive deformity of the foot that prevented application of the testing apparatus, open skin lesions, or an inability to walk unaided. On the basis of these criteria, one subject was excluded from analysis owing to an excessively unsteady gait. The sample, therefore, included $10 \mathrm{sub}-$ jects ( 9 women and 1 man) and 20 feet. Patient age ranged from 32 to 79 years (mean, 61 years), patient weight was 50 to $79 \mathrm{~kg}$ (mean, $69 \mathrm{~kg}$ ), and time since diagnosis was 4 to 34 years (mean, 15 years).

\section{Materials and Equipment}

Selection of the insoles and metatarsal pads (all from A. Algeo Ltd, Liverpool, England) was based on the fact that patients with rheumatoid arthritis benefit from a lower-density, softer material. ${ }^{15}$ Leber and Evanski ${ }^{24}$ found that latex reduced pressure under the metatarsal heads by as much as $28 \%\left(111 \mathrm{kN} / \mathrm{mm}^{2}\right)$. Two types of latex foam metatarsal pads were selected: a dome with a hardness of 29 Shore A (models SD2080 and SD2081; small and medium sizes) and a square bar of the same hardness (models SE1101, SE1102, and SE1103; small, medium, and large sizes). Different sizes were included to accommodate each individual foot size and shape. The prefabricated insole had a hardness of 25 Shore A and was made of 
expanded urethane foam (models UD1801, UD1802, and UD1803; small, medium, and large sizes).

The F-Scan in-shoe system (version 3.8; Tekscan, Boston, Massachusetts) was selected to measure forefoot plantar pressures; it permits measurement and analysis at high resolution and is therefore suitable for measurement of small anatomical sites, such as metatarsal regions. ${ }^{25}$ The system uses thin pressure sensors trimmed to fit the individual shoe with up to 960 sensor cells; sensors are linked to a computer via cables for data acquisition. Sampling of data and calibration of the sensors were performed in accordance with the manufacturer's instructions.

\section{Protocol}

Before data collection, plantar calluses were debrided in an attempt to maintain uniformity, as significant increases in plantar pressures have been recorded in their presence, ${ }^{26,27}$ although this was disputed in a recent study. ${ }^{28}$ The investigator (L.J.) selected the pad size on the basis of the depth of the transverse metatarsal arch and the width of the foot. Placement of the pads was determined by palpation of the metatarsal heads; the approximate center point of each metatarsal head was marked with a pen, and this image was then transferred to the dampened insole while the subject was weightbearing. Each pad was positioned $5 \mathrm{~mm}$ distal to the marked metatarsal heads in a central position using double-sided adhesive tape, thus creating the two insole designs shown in Figure 1. The three test conditions were shoe-only control, insole and dome pad, and insole and bar pad; subjects were randomly assigned and wore their own low-heeled, laced shoes with thin, 100\% nylon, ankle-high socks. The sensors were allowed time to warm up, an important procedure to control sensor variation by as much as $15 \%{ }^{29}$

This study adhered to the International Protocol Guidelines for Plantar Pressure Measurement, ${ }^{30}$ which state that no attempt should be made to control velocity because this may induce unnatural movement; gait, therefore, was not controlled in any way. Three separate walks along a straight, level corridor for each of the three conditions were measured. Subjects walked at their normal pace in a "figure eight" fashion. A final trial walk repeated the initial condition to enable comparative measurements of each sensor and its reliability. The steps were collected midcycle for data analysis, averaging three from each foot; the first and last steps were excluded to avoid acceleration and deceleration effects. ${ }^{31}$

The pilot study identified the need to refine the masking system used for data analysis. Three regions were identified based on the Win Masks software (Novel), in which percentages divide the foot into the first metatarsal; the second, third, and fourth metatarsals; and the fifth metatarsal. ${ }^{32,} 33$ The only adjustment made between subjects was to realign the top border of the mask up or down to fully capture the forefoot (Fig. 1).

\section{Statistical Analysis}

A one-way analysis of variance (ANOVA) $(\alpha=.05)$ was used to determine whether significant differences existed among the conditions, followed, as appropriate, by $t$-tests to establish where these differences existed. A Bonferroni adjustment for multiple testing was applied to the ANOVA and the $t$-tests.

\section{Results}

Both insole designs reduced mean peak pressures, with significant reductions at the central (second, third, and fourth) metatarsal heads $(P<.02)$ (Fig. 2).

Compared with the shoe-only control condition, average reductions in mean peak pressure of $13.5 \%$ $(19 \mathrm{kPa})$ for the insole and dome pad design and 22.6\% (32 $\mathrm{kPa}$ ) for the insole and bar pad design were found over the first metatarsal, but these reductions were not statistically significant. The central metatarsal mean peak pressure was reduced by $11.8 \%(33 \mathrm{kPa})$ in the insole and dome pad condition and by $21.3 \%(58 \mathrm{kPa})$ in the insole and bar pad condition compared with the shoe-only control condition $(P<.02)$. Mean peak plantar pressure also decreased over the fifth metatarsal: $9 \%(5 \mathrm{kPa})$ and $26.4 \%(16 \mathrm{kPa})$ in the insole and dome pad and insole and bar pad conditions, respectively. However, these reductions were not statistically significant.

A significant difference was demonstrated over the central metatarsals between the two insoles favoring the insole and bar pad design $(P<.02)$. Pressure data for the central metatarsal head region for each condition are given in Table 1.

To determine more fully the effect of the insoles over the central metatarsals, the pressure variables listed in Table 1 detail the decrease in the pressuretime integral for each experimental condition. There was a significant difference between the insole and bar pad design and the shoe-only control condition $(P<.05)$. Stance times and contact areas increased in the insole and dome pad and, to a greater extent, insole and bar pad conditions, although not in a statistically significant manner. Individual subject analysis revealed a maximum reduction in peak pressure of $35 \%$ over the central metatarsal region when using 

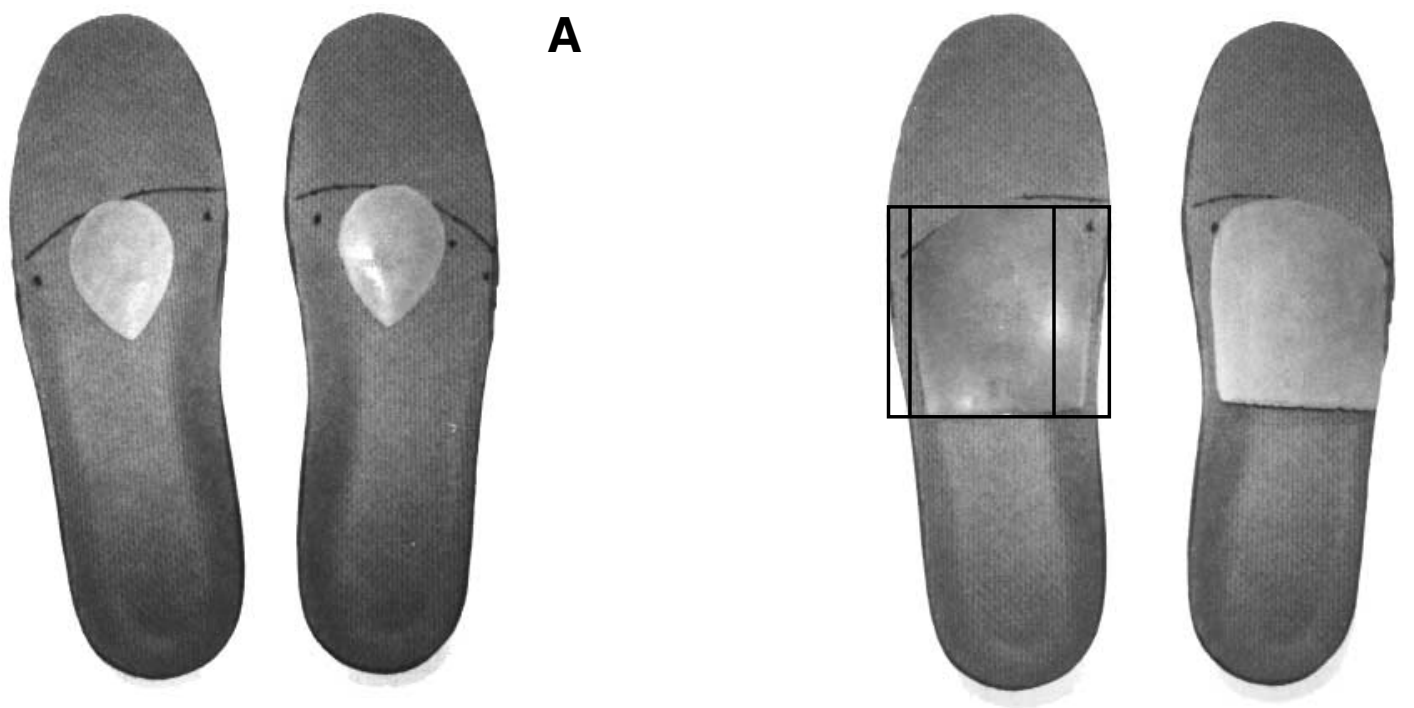

B

Figure 1. Prefabricated insoles and metatarsal padding: insole and dome pad (A) and insole and bar pad (B) conditions. The boxed region illustrates an approximation of the F-Scan masking used.

the insole and bar pad design compared with the shoe-only condition; the minimum change in peak pressure was $4 \%$ when using the insole and dome pad design.

No significant differences in mean cadence were noted among conditions using a one-way ANOVA ( shoe-only condition $=1.683 \mathrm{sec}$, insole and dome

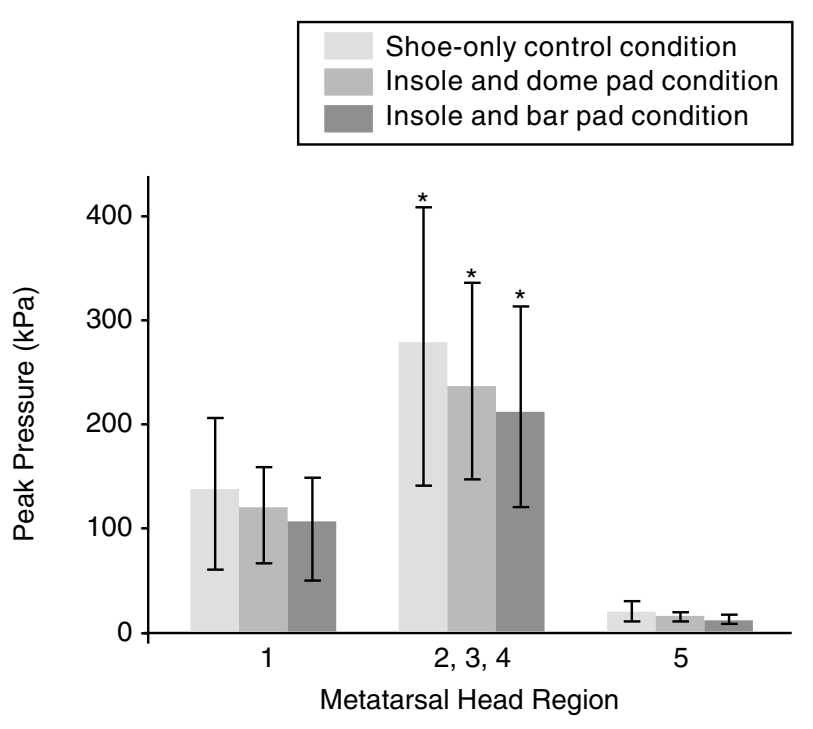

Figure 2. Mean peak pressures for each condition over the three designated foot regions. Error bars indicate SD. *Significant differences $(P<.02)$ identified using $t$-tests. pad condition $=1.696 \mathrm{sec}$, and insole and bar pad condition $=1.701 \mathrm{sec} ; \mathrm{F}=0.10 ; P=.95$ ). Cadence was calculated by dividing the number of steps by the time from initial contact to final heel contact (left and right steps). Cadence values were averaged from the trials to give the cadence score for each condition.

When the first and last trials were compared, the overall repeatability of the sensors was found to be good $(r=0.96 ; P=.10)$. When evaluating each sensor individually, changes were noted that ranged from a $4 \%$ decrease to a $9 \%$ increase in pressure.

Of the ten subjects, seven preferred the insole and dome pad design and three chose the insole and bar pad design. The chosen insole was issued to the subject on completion of the study.

\section{Discussion}

The results of this study demonstrate that prefabricated insoles incorporating metatarsal padding can be an effective treatment for high forefoot plantar pressures in patients with rheumatoid arthritis. The results show that there is a significant difference between the two insole designs. The central metatarsals were selected for further analysis, as this area is often the focal point of pain.1,15,34-36 The study by Minns and Craxford ${ }^{35}$ found that pressures under the metatarsal heads in subjects with rheumatoid arthritis were two and three times those in healthy subjects. All of the subjects benefited from reduced plantar pressures with each insole design; in only one subject were the differences negligible between 


\begin{tabular}{llcc}
\hline \multicolumn{2}{l}{ Table 1. Pressure Data for the Central Metatarsal Head Region by Condition } \\
\hline & \multicolumn{1}{c}{ Condition } \\
\cline { 2 - 4 } Variable & \multicolumn{1}{c}{ Shoe Only } & Insole and Dome Pad & Insole and Bar Pad \\
\hline Pressure-time integral, mean (SD) (kPa sec) & $100.6(25.1)$ & $96.3(20.8)$ & $87.7(21.4)^{a}$ \\
Peak pressure, mean (SD) (kPa) & $274.5(49.3)$ & $242.0(40.0)^{a}$ & $216.1(46.6)^{a}$ \\
Stance time, mean (range) (sec) & $0.758(0.673-0.864)$ & $0.788(0.693-0.879)$ & $0.790(0.669-0.935)$ \\
Contact area, mean (range) $\left(\mathrm{cm}^{2}\right)$ & $125.4(90.6-148.7)$ & $138.5(101.0-161.1)$ & $144.1(106.0-170.6)$ \\
\hline
\end{tabular}

aSignificant differences $(P<.05)$ identified using $t$-tests.

the two insoles. An average decrease in the pressure-time integral with both insoles over the central metatarsals provides additional evidence that there was a reduction in sustained pressure with the insole and bar pad design compared with the shoe-only control condition.

Contact time is closely related to a variation in gait and has been demonstrated to affect plantar pressure ${ }^{37}$ The contact time changes seen in this study between conditions were minimal and not significant, although contact time did increase slightly in each experimental condition. A key purpose of plantar padding is to provide a means of increasing contact area and unloading focal points of high pressure. As expected, contact area increased with both insoles. ${ }^{14,15}$

When Hodge et al ${ }^{15}$ investigated orthotic management of forefoot plantar pressure in rheumatoid arthritis, a low-density, prefabricated foot orthosis without metatarsal padding was found to be as effective at reducing plantar pressures as a custom-molded foot orthosis with metatarsal padding. Hodge et al concluded that metatarsal domes and bars (a boomerang-shaped device) were equally effective in reducing metatarsal head pressures. The present study also found that each type of pad offers effective management, but the bar design selected in this study performed better than the dome design, which may be attributable to the increased width and length of this device. The insole preferred by most subjects was the dome pad design, although the recommended insole was the bar pad design. It is well known that dorsal sites are often vulnerable in this patient group, ${ }^{1,3-5}$ particularly when there is insufficient toe box provision in shoes, and it is postulated that the insole and bar pad design created too much bulk in certain instances with inadequate footwear. Any increase or decrease in discomfort experienced by the subjects was not investigated in this research, and thus no statistical relationship among pain, pressure, and footwear was considered. Footwear will always dictate the treatment options available, but, where possible, the insole and bar pad design is the preferred choice in first-line management. When footwear is too restrictive, the insole and dome pad design offers a good alternative intervention. This study did not analyze the whole foot, and so pressure increases might have occurred at adjoining sites. ${ }^{13,19,38}$

The equipment used in this study has had a mixed reception regarding its accuracy as a clinical tool, ${ }^{39-42}$ with reports of variations between sensors, wear, excessive bending, and sensor degradation over time. In this study, sensors were reused between subjects; as there were ten subjects and each walked a total of ten trials, there was concern regarding sensor reliability with continued use. For this reason, the study provided a means of assessing the output from each sensor, comparing the first and last trials using the same condition. Any discernible drop in pressure would have become apparent at the end of each test session, but no significant change was noted overall. The results of this study, therefore, demonstrate that the sensors used were reliable, and it is recommended that this procedure be used for F-Scan measurements to ensure reliability of results. Analysis of specific regions of the foot necessitates a system of masking; with each individual foot displaying different degrees of anatomical variation, this has proved to be a contentious issue with various masking methods reported. $.^{7,},{ }^{13}$ It is essential that there is consistent identification of the chosen sites ${ }^{30}$ and this remains subject to individual interpretation and an inherent weakness in plantar pressure analysis. Although there are drawbacks with most plantar pressure measurement systems, the best indices for reliability of the F-Scan system are the peak pressures over the metatarsal heads. ${ }^{43,44}$

Clinical evidence is required in order to formulate a national framework for the treatment of rheumatoid arthritis, ${ }^{45}$ and it is hoped that this study will provide the impetus to establish a protocol for the management of high plantar pressures in established rheumatoid arthritis. There is an opportunity for further research in plantar pressure management in 
rheumatoid arthritis that investigates a broader range of materials and metatarsal pad shapes.

\section{Conclusion}

As a first-line treatment in rheumatoid arthritis, it is recommended that a bar pad made of latex or a similar material be placed approximately $5 \mathrm{~mm}$ distal to the center of the metatarsal heads and incorporated into a low-density insole. In cases in which footwear may prove too restrictive, a dome design should be used. Prefabricated devices offer an effective, rapid, and relatively low cost treatment for the reduction of plantar pressures in established rheumatoid arthritis.

Acknowledgment. James Sheridan of A. Algeo Ltd, Liverpool, England, for providing the insoles for this study.

\section{References}

1. Woodburn J, West SG: "Pain in the Foot," in $A B C$ of Rheumatology, ed by ML Snaith, p 18, BMJ Publishing Group, London, 1999.

2. Chalmers AC, Busby C, Goyert J, et Al: Metatarsalgia and rheumatoid arthritis: a randomized, single blind, sequential trial comparing 2 types of foot orthoses and supportive shoes. J Rheumatol 27: 1643, 2000.

3. Kerry RM, Holt GM, Stockley I: The foot in chronic rheumatoid arthritis: a continuing problem. The Foot 4: 201, 1994.

4. Woodburn J, Helliwell PS: Foot problems in rheumatology. Br J Rheumatol 36: 932, 1997.

5. O'Connell PG, Lohmann Siegel K, Kepple TM, et al: Forefoot deformity, pain and mobility in rheumatoid and nonarthritic subjects. J Rheumatol 25: 1681, 1998.

6. Burra G, Katchis SD: Rheumatoid arthritis of the forefoot. Clin Podiatr Med Surg 16: 363, 1999.

7. Wiener-Ogilvie S: The foot in rheumatoid arthritis. The Foot 9: 169, 1999.

8. Craxford AD, Stevens J, Park C: Management of the deformed rheumatoid forefoot: a comparison of conservative and surgical methods. Clin Orthop (June): 121, 1982.

9. Woodburn J, Helliwell PS: Relation between heel position and the distribution of forefoot plantar pressures and skin callosities in rheumatoid arthritis. Ann Rheum Dis 55: 806, 1996.

10. Veves A, Hay EM, Boulton AJM: The use of padded hosiery in the painful rheumatoid foot. The Foot 1: 175, 1992.

11. Shi K, Tomita T, Hayashida K, et al: Foot deformities in rheumatoid arthritis and relevance of disease severity. J Rheumatol 27: 84, 2000.

12. SHRADER JA: Nonsurgical management of the foot and ankle affected by rheumatoid arthritis. J Orthop Sports Phys Ther 29: 703, 1999.

13. MERRITT JL: Advances in orthotics for the patient with rheumatoid arthritis. J Rheumatol 14 (suppl 15): 62, 1987.

14. White SC: "Padding and Taping Techniques," in Clini- cal Biomechanics of the Lower Extremities, ed by RL Valmassy, p 375, CV Mosby, St Louis, 1996.

15. Hodge MC, BACH TM, CARTER GM: Orthotic management of plantar pressure and pain in rheumatoid arthritis. Clin Biomech 14: 567, 1999.

16. YATES B: A national audit of appliance practices in podiatry. J Br Podiatr Med 51: 120, 1996.

17. Pratt DJ: A critical review of the literature on foot orthoses. JAPMA 90: 339, 2000.

18. Holmes GB, Timmerman L: A quantitative assessment of the effect of metatarsal pads on plantar pressures. Foot Ankle 11: 141, 1990.

19. Chang A, Abu-Faraj ZU, Harris GF, et AL: Multistep measurement of plantar pressure alterations using metatarsal pads. Foot Ankle Int 15: 654, 1994.

20. Hayda R, Tremaine MD, Tremaine K, et al: Effect of metatarsal pads and their positioning: a quantitative assessment. Foot Ankle Int 15: 561, 1994.

21. Mclauchlan PT, Abboud RJ, Rendall GC, et al: Use of an in-shoe pressure system to investigate the effect of two clinical treatment methods for metatarsalgia. The Foot 4: 204, 1994.

22. Kelly A, Winson I: Use of ready-made insoles in the treatment of lesser metatarsalgia: a prospective randomised controlled trial. Foot Ankle Int 19: 217, 1998.

23. Schumacher HR JR, Klippel JH, Koopman WS (eds): Primer on the Rheumatic Diseases, 10th Ed, Arthritis Foundation, Atlanta, 1993.

24. Leber C, Evanski PM: A comparison of shoe insole materials in plantar pressure relief. Prosthet Orthot Int 10: $135,1986$.

25. Woodburn J, Helliwell PS: Observations on the F-Scan in-shoe pressure measuring system. Clin Biomech 11: 301, 1996.

26. Young MJ, Cavanagh PR, Thomas G, et al: The effect of callus removal on dynamic plantar foot pressures in diabetic patients. Diabet Med 9: 55, 1992.

27. Pitei DL, Foster A, Edmonds M: The effect of regular callus removal on foot pressures. J Foot Ankle Surg 38: 251, 1999.

28. Woodburn J, Stableford Z, Helliwell PS: Preliminary investigation of debridement of plantar callosities in rheumatoid arthritis. Rheumatology 39: 652, 2000.

29. Mueller MJ, Strube MJ: Generalizability of in-shoe peak pressure measures using the F-scan system. Clin Biomech 11: 159, 1996.

30. Barnett S, for the Foot Pressure Interest Group: International protocol guidelines for plantar pressure measurement. The Diabetic Foot 1: 137, 1998.

31. Cavanagh PR, Hewitt FG Jr, Perry JE: In-shoe plantar pressure measurement: a review. The Foot 2: 185, 1992.

32. Cornwall MW, McPoIl TG: Effect of foot orthotics on the initiation of plantar surface loading. The Foot 7: 148, 1997.

33. Wearing SC, Urry S, Smeathers JE, et al: A comparison of gait initiation and termination methods for obtaining plantar foot pressures. Gait Posture 10: 255, 1999.

34. Gould J: Conservative management of the hypersensitive foot in rheumatoid arthritis. Foot Ankle 2: 224, 1982.

35. Minns RJ, CRAXFord AD: Pressure under the forefoot in rheumatoid arthritis: a comparison of static and dynamic methods of assessment. Clin Orthop (Jul-Aug): 235, 1984.

36. Postema K, Burm PE, Zande ME, et al: Primary meta- 
tarsalgia: the influence of a custom moulded insole and a rockerbar on plantar pressure. Prosthet Orthot Int 22: 35, 1998.

37. Hautmann S, Rosenbaum D, Gold M, et al: On the importance of monitoring walking speed in pressure distribution measurements. Gait Posture 2: 250, 1994.

38. Schaff PS, Cavanagh PR: Shoes for the insensitive foot: the effect of a "rocker bottom" shoe modification on plantar pressure distribution. Foot Ankle 11: 129, 1990.

39. LuO ZP, BERGLUnd LJ, AN KN: Validation of F-Scan pressure sensor system: a technical note. J Rehabil Res Dev 35: 186, 1998.

40. Sumiya T, Suzuki Y, Kasahara T, et al: Sensing stability and dynamic response of the F-Scan in-shoe sensing system: a technical note. J Rehabil Res Dev 35: 192, 1998.
41. Nicolopoulos CS, Anderson EG, Solomonidis SE, ET aL: Evaluation of the gait analysis FSCAN pressure system: clinical tool or toy? The Foot 10: 124, 2000.

42. WeSt S, BARNETT S: Plantar pressure measurement: which system? The Diabetic Foot 2: 108, 1999.

43. Brown M, Rudicel S, Esquenazi A: Measurement of dynamic pressures at the shoe-foot interface during normal walking with various foot orthoses using the FSCAN system. Foot Ankle Int 17: 152, 1996.

44. Ahroni JH, Boyko EJ, Forsberg R: Reliability of F-Scan in-shoe measurements of plantar pressure. Foot Ankle Int 19: 668, 1998.

45. Department of Health: The New NHS, Modern and Dependable: A National Framework for Assessing Performance, Her Majesty's Stationery Office, London, 1998.

\section{ERRATUM}

In "Rheumatologic Conditions of the Foot" by Michelle Costa, DO, Tina Rizak, MD, MPH, and Bernard Zimmermann, MD, March/April 2004, page 177, the second author's last name was misspelled. The correct spelling is Rizack. 